\title{
Exemples concrets d'annonce des crues dans de petits bassins versants
}

\author{
Concrete examples of flood-forecasting methods \\ for small catchment areas
}

Jean-Michel Dujardin

SOGREAH

\section{Conception d'un système d'annonce des crues}

Les systèmes de prévision de crues ont pour objet de prévoir, à partir des seules données disponibles au temps initial, l'évolution de la crue d'un bassin versant dans les quelques heures à venir, et d'avertir les services concernés avec une avance suffisante pour prendre les mesures protectrices nécessaires en cas de risque de débordement.

Le système d'annonce de crues comporte :

- une ou plusieurs méthodes de calcul qui constituent le modèle,

- un réseau de stations de mesure qui relève les données à fournir au modèle,

- un système de transmission pour acheminer les mesures au centre de calcul dans un délai court compatible avec l'avance souhaitée,

- une procédure d'exploitation définissant les règles de fonctionnement et les responsabilités de chaque personnel concerné (vigilance, alerte, calcul, diffusion des résultats).

Le modèle d'annonce de crues ne peut être opérationnel que si l'ensemble du système (moyens de calcul + station de mesure + transmissions) est conçu de façon cohérente, et que si tous les rouages fonctionnent.

Un modèle d'annonce de crues comporte deux fonctions essentielles :

- une fonction de transformation de l'eau pluviale disponible à la surface d'une aire élémentaire en écoulement à la sortie de cette aire (modèle de transformation pluie-débit);

- une fonction de propagation et de composition dans le réseau hydrographique des écoulements foumis par les aires élémentaires (modèles de flood-routing). On peut éventuellement $y$ ajouter une simulation $d u$ fonctionnement des ouvrages hydrauliques et de leur action sur les écoulements naturels (champ d'inondation, dérivations, retenues, etc.).
On peut distinguer trois types de modèles de prévision selon que l'une, l'autre, ou les deux fonctions indiquées ci-dessus seront prises en compte. Le choix entre ces trois types de modèles dépendra de la taille et des caractéristiques du bassin versant à étudier.

\section{Annonce des crues dans les petits bassins versants}

Nous nous intéressons ici aux systèmes d'annonce de crue basés sur une transformation directe de la pluie en débit à l'exutoire et nous présentons les deux méthodes que nous avons utilisées.

Elles ont ceci de commun qu'elles font appel toutes deux à la notion de coefficient de ruissellement attaché à l'averse génératrice de la crue et que ce coefficient de ruissellement est très fortement variable en fonction des conditions initiales ou des pluies antérieures.

Elles ont également en commun un objectif de précision raisonnable mais limité. Etant donné une incertitude inévitable dans l'estimation de la pluie moyenne qui affecte le bassin versant (on utilise pour cela deux ou trois postes correctement répartis), une schématisation assez sommaire du processus de transformation pluiedébit a paru raisonnable et se justifie par les résultats obtenus.

Dans ces conditions, ces méthodes permettent d'abord de détecter de façon systématique les périodes de vigilance pendant lesquelles il y a un risque de crue évident.

Ensuite, lorsqu'une crue se déclenche effectivement, elles permettent de savoir assez vite s'il s'agira d'une crue ordinaire, forte, très forte, extraordinaire, et d'indiquer avec une certaine antériorité si le maximum est en voie d'être atteint, ou si une aggravation est encore prévisible. Par contre, elles restent relativement imprécises sur la valeur du débit de pointe effectivement atteint et sur l'heure exacte de réalisation de ce maximum. 
Elles constituent, à notre sens, un outil simple et opérationnel permettant de répondre à certaines préoccupations immédiates du prévisionniste et des populations. Mais ce ne devrait être qu'un premier pas vers un outil plus perfectionné dont la réalisation nécessiterait des données d'observations plus détaillées et portant sur une période assez longue.

Selon la taille du bassin, ou bien on découpe la crue en plusieurs pas de temps par une méthode discrète, ou bien on la considère de façon globale.

\section{Méthode discrète - modèle Prévik}

Sur les bassins de 500 à $2000 \mathrm{~km}^{2}$, la crue à reconstituer est découpée en pas de temps. Le choix du pas de temps, en général de quatre heures à dix-huit heures, est fonction du temps de concentration du bassin.

La transformation pluie-débit s'effectue en deux opérations :

- Transformation de la précipitation en pluie nette, disponible pour le ruissellement par une fonction de production ;

- Déformation de la pluie nette en hydrogramme à l'exutoire du bassin par une fonction d'étalement dans le temps.

\section{Fonction production}

La pluie nette $R j$, associée à chaque pas de temps à la précipitation $P j$ est calculée par l'intermédiaire du coefficient de ruissellement $C j$.

Le coefficient de ruissellement à appliquer à la précipitation est relié à l'état de saturation du sol dû aux précipitations antérieures. On peut suivre son évolution à chaque pas de temps par la formule de récurrence :

dans laquelle :

$$
I_{j+1}=k \cdot\left(I_{j}+P_{j}\right) \leqslant I_{\max }
$$

$k$ est un coefficient inférieur à 1 et lié au pas de temps

$I_{\max }$ est la borne supérieure de l'indice de saturation.

On exprime, par une opération de réglage, la relation généralement linéaire entre $I j$ et $C j$ et l'on calcule la pluie nette :

$$
R_{j}=\left(a I_{j}+b\right) P_{j}
$$

Au départ d'une crue, il n'est pas commode de reconstituer l'historique des pluies du passé, mais il y a souvent une relation étroite entre la valeur initiale de $I$ et le débit de base de la rivière. La saturation initiale sera calculée à partir du débit de la rivière au moment où débute la crue :

$$
I_{0}=f\left(Q_{0}\right)
$$

\section{Fonction modulation}

La pluie nette du pas de temps $J$ se répartit en écoulements au cours des pas de temps successifs :

$$
\begin{aligned}
& x R_{j} \text { pendant le pas de temps } J+1 \\
& y R_{j} \text { pendant le pas de temps } J+2
\end{aligned}
$$

suivis d'une décrue de coefficient de tarissement $\alpha$, soit à chaque pas de temps successif $\alpha y R_{j}, \alpha^{2} y . R_{j}$, etc...

La somme de tous ces écoulements étant égale à $R_{j}$ on aura $y=(1-\alpha)(1-x)$ et la fonction modulation comporte deux paramètres.

La lame d'eau écoulée pendant chaque pas de temps est obtenue en sommant les écoulements dus à toutes les pluies élémentaires et l'on aura finalement:

$$
Q_{j+1}=\alpha \cdot Q_{j}+x R_{j}+(1-\alpha-x) R_{j-1}
$$

\section{Application de la méthode}

En suivant en permanence le débit de la rivière à la station limnimétrique pour laquelle on effectue des prévisions, on peut contrôler l'évolution de $I_{0}=f\left(Q_{0}\right)$ et savoir à partir de quel seuil le coefficient de ruissellement est susceptible de transformer une certaine pluie potentielle en crue.

Au démarrage d'une crue on suit son évolution, réactualisée à chaque pas de temps, selon les formules indiquées. On utilise cependant pour le terme $Q_{j}$ la valeur trouvée lors de la prévision précédente et non pas la valeur observée au temps $j$ à la station limnimétrique.

La prévision permet une avance de un à deux pas de temps sur la réalisation de la crue selon la valeur de $x$ et le poids relatif de $P_{j-1}$ et $P_{j}$.

\section{Exemple d'application - la Gartempe à Montmorillon}

Le bassin versant a une superficie de $1875 \mathrm{~km}^{2}$. Les crues provenant de l'amont sont fortement modifiées et accélérées par les affluents aval et les observations de niveau sur le réseau hydrographique sont inopérantes pour prévoir les crues à Montmorillon.

On a utilisé la méthode Prévik avec un pas de temps de six heures. La pluie moyenne en six heures est calculée à l'aide de quatre stations pluviométriques réparties dans le bassin. Le réglage du modèle se fait par simulation des crues du passé.

Les équations de la prévision sont :

$$
\begin{aligned}
& I_{0}=2,5 Q_{0}^{0,60} \\
& I_{j+1}=0,97\left(I_{j}+P_{j}\right) \leqslant 60 \\
& R_{j}=0,0125 I_{j} P_{j} \\
& Q_{j+1}=0,85 Q_{j}+0,15 R_{j}
\end{aligned}
$$

La méthode procure une avance de six à douze heures et permet notamment de détecter assez vite si la crue atteindra ou non le seuil de débordement de $220 \mathrm{~m} / \mathrm{s}$. Sur les vingt tet-une crues simulées, on a obtenu les écarts suivants entre débits prévus et réalisés :

- inférieurs à $10 \%$ pour neuf crues,

- de $10 \%$ à $20 \%$ pour huit crues,

- de $20 \%$ à $30 \%$ pour quatre crues.

Le problème de la précision est surtout important au voisinage du seuil de débordement : toutes les crues prévues, supérieures à $250 \mathrm{~m}^{3} / \mathrm{s}$, ont effectivement débordé ; toutes les crues prévues, inférieures à $200 \mathrm{~m}^{3} / \mathrm{s}$, sont effectivement passées sous le seuil de débordement. 

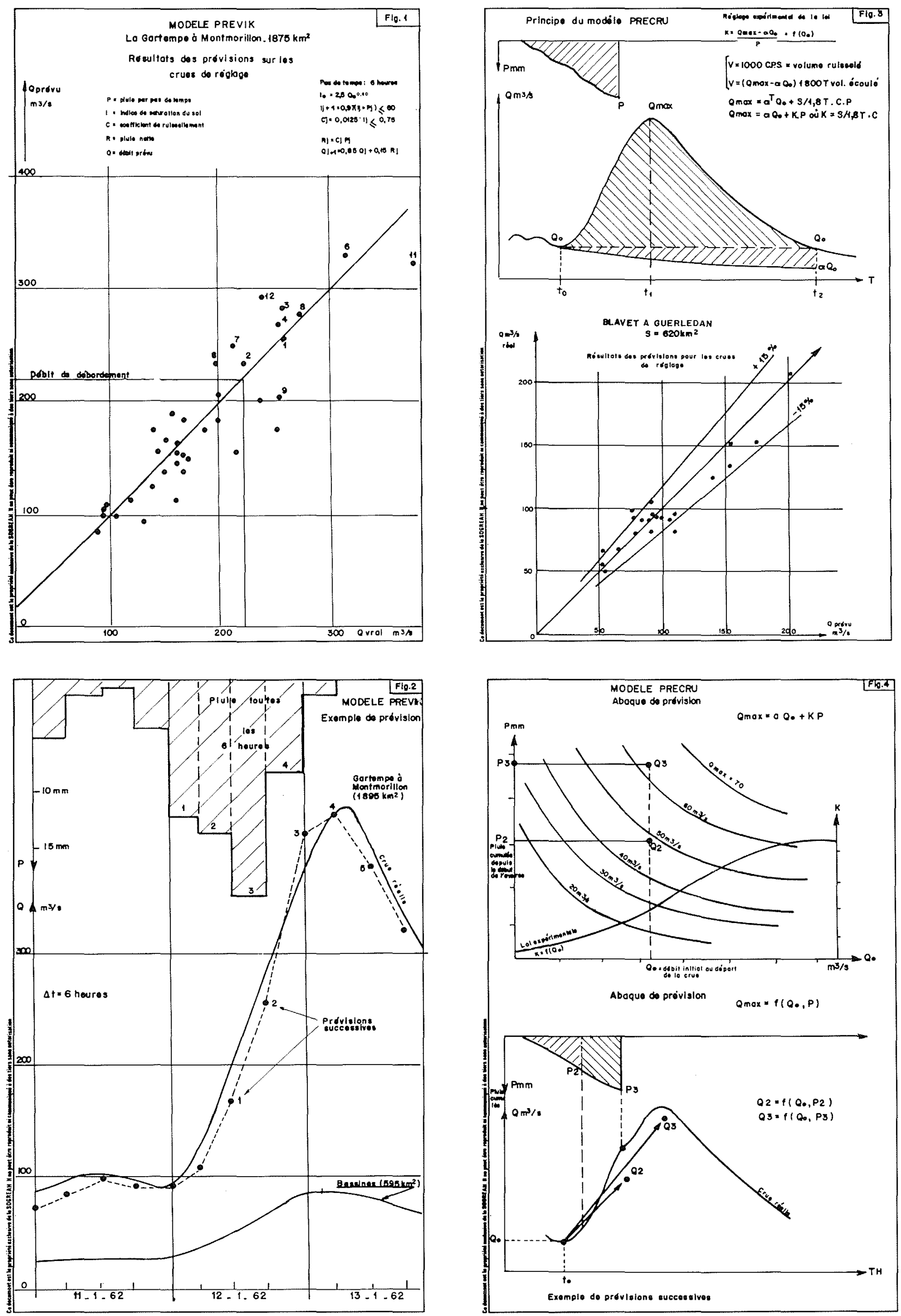
Pour les crues prévues entre 200 et $250 \mathrm{~m}^{3} / \mathrm{s}$ on ne peut se prononcer qu'au dernier moment, mais le débordement éventuel est en tous cas très faible.

\section{Méthode globale - modèle Précru}

Sur les bassins très petits, il n'est pas toujours possible ou efficace de découper la crue en pas de temps réguliers et on utilise une méthode permettant de prévoir directement le débit de pointe de crue à partir du débit initial de la rivière, du volume total de l'averse et de sa durée.

\section{Formulation de la méthode}

Une pluie de Pmm tombant sur un bassin de superficie $S \mathrm{~km}^{2}$ produira un volume de ruissellement :

$$
V \mathrm{~m}^{3}=1000 \text {.C.P.S }
$$

où $C$ est le coefficient de ruissellement moyen de l'averse.

Au démarrage de la crue, la rivière a un débit initial $Q_{0}$ et l'on admet qu'en l'absence de pluie ce débit aurait suivi une courbe de décroissance exponentielle $Q=Q_{0} \alpha^{t}$.

L'averse $P$ provoque une crue qui atteint son maximum $Q_{\max }$ après un temps de montée $t_{1}$, décroît pour revenir au débit $Q_{0}$ après un temps de descente $t_{2}$ et a un temps de base $T=t_{1}+t_{2}$.

En assimilant la crue à un triangle, ou à tout hydrogramme type de même volume, on aura comme volume ruisselé au-dessus de la courbe de décroissance :

$$
V \mathrm{~m}^{3}=\left(Q_{\max }-\alpha^{T} Q_{0}\right) \times 1000 T
$$

En admettant : volume ruisselé = volume écoulé, on aura:

$$
Q_{\max }=\alpha^{T} Q_{0}+\frac{S}{1,8 T} \cdot \mathrm{C} . \mathrm{P}
$$

En première approximation on admet que le temps de base des crues est constant et que c'est une caractéristique du bassin versant. La formule devient :

$$
Q_{\max }=a Q_{0}+K P
$$

où $K$ est proportionnel au coefficient de ruissellement de. l'averse considérée.

Le coefficient $K$ est fonction de l'état de saturation du sol au moment où se produit l'averse et peut être rattaché à un indice de précipitations antérieures ou même directement au débit initial $Q_{0}$ considéré lui-même comme un indice des pluies antérieures.

Le réglage du modèle consiste à établir expérimentalement, par l'analyse des crues du passé, une loi $K=f\left(Q_{0}\right)$. On peut ensuite, éventuellement, affiner la méthode en prenant en compte la durée $t_{a}$ de l'averse génératrice et son incidence sur le temps de base de la crue, ce qui se traduit par l'établissement d'une loi :

$$
K=\frac{a}{b+t_{a}} \cdot f\left(Q_{0}\right)
$$

La méthode se concrétise par l'établissement d'un abaque sur lequel on lit directement $Q_{\max }$ en fonction de $Q_{0}$ et $P$. On peut établir un abaque similaire pour le volume de la crue si c'est la variable utile de la prévision (cas d'un barrage).

\section{Utilisation de la méthode}

On peut suivre en permanence l'évolution de la valeur de $K$ en fonction du débit de la rivière et définir un seuil de vigilance lorsque $K$ atteint une valeur telle qu'une pluie de moyenne importance suffirait à provoquer une crue débordante.

Lorsqu'une crue se déclenche, on note la valeur $K=f\left(Q_{0}\right)$ qui restera valable pour toute la crue, on suit l'évolution de $P$ qui est le volume pluvial cumulé depuis le début de l'averse et on actualise en conséquence le débit de pointe prévisible.

On peut éventuellement prendre comme valeur de $P$ non seulement la pluie effectivement mesurée sur le ou les postes pluviométriques représentatifs, mais y ajouter le volume pluvial prévisible si la météorologie est en mesure de prévoir une prolongation de l'épisode pluvieux en cours.

La principale difficulté rencontrée réside dans la représentativité du débit initial comme indice de saturation. Dans certains cas, on a dû avoir recours à un indice des pluies antérieur et élaborer une relation $K=f(I)$; dans d'autres, on assiste à des crues successives sans retour au débit de base et l'on est obligé de revenir à la méthode de discrétisation en pas de temps, mais, pour les petits bassins, la méthode globale est pratique et satisfaisante.

\section{Exemples d'application}

\section{Ville de Morlaix}

Bassin de $200 \mathrm{~km}^{2}$ composé de deux bassins partiels qui convergent à l'entrée de la ville, Prévision basée sur deux stations limnimétriques et un pluviophone. Débit de débordement : $30 \mathrm{~m}^{3} / \mathrm{s}$. Prévision avec quatre à six heures d'avance de toute crue supérieure à $15 \mathrm{~m}^{3} / \mathrm{s}$, avec une précision de $\pm 15 \%$.

\section{Ville de Quimper}

Bassin de $500 \mathrm{~km}^{2}$ composé de trois bassins partiels qui convergent à Quimper. Prévision basée sur deux stations limnimétriques et trois pluviophones. Débit admissible de l'Odet à Quimper : $65 \mathrm{~m}^{3} / \mathrm{s}$. Sur un débit initial de $30 \mathrm{~m}^{3} / \mathrm{s}$ il faut une averse de $30 \mathrm{~mm}$ pour atteindre $60 \mathrm{~m}^{3} / \mathrm{s}$ ce qui situe le seuil de vigilance. La prévision donne une avance de six heures à dix heures sur les crues à Quimper avec une précision de $\pm 10 \mathrm{~m}^{3} / \mathrm{s}$.

\section{Le Blavet à Guerlédan et Pontivy}

Bassin de $620 \mathrm{~km}^{2}$ au barrage de Guerlédan et de $880 \mathrm{~km}^{2}$ à Pontivy. Prévision à l'aide de quatre postes pluviographiques et une station limnimétrique. Avance de douze heures à quinze heures sur la crue entrant dans la retenue de Guerlédan dont on prévoit le volume. Prévisions des crues à Pontivy avec seize heures à dixhuit heures d'avance et une estimation du débit de pointe à $\pm 15 \%$ près. 
Cette étude avait pour but complémentaire de proposer de nouvelles consignes d'exploitation du barrage du Guerlédan permettant de concilier son rôle énergétique avec l'écrêtement des crues.

\section{Conditions rencontrées pour les cas pratiques de mise en œuvre}

Généralement, le problème de l'élaboration d'une procédure d'annonce des crues sur de petits bassins nous est posé dans des cas où il n'existait jusque là aucune prévision et très peu d'observations disponibles, la rapidité du temps de réponse du bassin ayant paru décourager toute tentative.
A partir des seules données existantes, et pour un travail de mise au point très raisonnable, ces méthodes permettent d'élaborer un modèle de prévision opérationnel mais perfectible, la similation des crues du passé laissant bien augurer des performances escomptables. Sa mise en cuvre effective reste conditionnée par l'implantation adéquate d'une ou plusieurs stations pluviométriques à transmission rapide (pluviophones par exemple).

Les études que nous avons réalisées dans ce domaine sont encore trop récentes pour que nous puissions juger de leur efficacité opérationnelle et examiner si des mesures d'observations nouvelles et plus appropriées permettraient d'améliorer leurs performances.

\section{Discussion}

Le Président. - Je vous remercie de nous avoir montré, sans résoudre bien sûr le problème complet de la prévision des hydrogrammes de crues, comment définir des indicateurs de façon séquentielle.

Vous vous demandiez si l'on peut aller plus loin, vers une prévision plus complète, en tenant compte de tous les éléments que vous avez donnés. Je rappelle que cela a été fait à Périgueux pour l'annonce des crues de l'Isle et de la Vézère, en utilisant des fonctions de transfert tout à fait expérimentales puisqu'elles sont déduites de l'analyse des données de crues antérieures. En ce qui concerne l'utilisation des prévisions météorologiques, le centre de prévision des crues de Périgueux, en liaison avec la Météorologie de Bordeaux, parvient également à intégrer assez bien les risques de précipitations importantes sur les bassins surveillés. D'autre part, pour la distribution spatiale des précipitations, le centre de Périgueux dispose des informations d'un radar qui suit dans le temps l'évolution, les déplacements des épicentres de précipitations.

Enfin je remarque que le schéma que vous avez utilisé est exactement celui retenu sur le bassin expérimental d'Alrance pour définir une relation pluie-débit valable sur de petits bassins à dominante imperméable.

J'ouvre maintenant la discussion.

$M$. DUJARDIN. - Je pense que le progrès, dans ce domaine, sera une œuvre collective. Il faut que chacun apporte, comme aujourd'hui, sa contribution, même partielle, au problème : ceux qui s'intéressent au problème des transmissions, ceux qui s'intéressent à la représentativité d'une pluie moyenne sur un bassin, à la répartition spatiale des pluies.

Il faut aussi que les chercheurs perçoivent les difficultés réelles de l'utilisateur sur place. J'ai appris beaucoup, sur le ter- rain, au contact des responsables de la prévision et de leurs problèmes. Il ne s'agit pas de mathématique ou de modèle, il s'agit, le moment venu, de prendre une décision.

M. CAZENAVE. - Ce que vient de nous dire M. DUJARDIN, c'est précisément le rôle de la Société Hydrotechnique de France et de ses Groupes de travail. Nous travaillons très activement à cette rencontre entre les utilisateurs et les théoriciens. Il y a là un cadre qui satisfait pleinement notre but.

Le Président. - Je voudrais faire une remarque à propos des comparaisons entre observations et prévisions. Il faut bien préciser si ces comparaisons portent sur des événements (crues) qui ont servi à l'étalonnage du modèle ou, au contraire, sur des événements observés et calculés non pris en compte dans l'ajustement. Seule cette dernière relation permet de vérifier la valeur "prédictive" du modèle en vue de son utilisation opérationnelle.

M. DUJARDIN. - Sur la précision des outils que je vous ai présentés, il y a deux choses à dire :

- D'abord, pour mettre en cuvre le système, il faut installer les transmissions, c'est-à-dire qu'il s'écoule un certain délai entre le moment où la méthode est instaurée et celui où elle devient opérationnelle. Les études réalisées sont trop récentes pour que j'aie des renseignements sur leur efficacité pratique. - Ensuite au niveau de la comparaison entre observations et prévisions, qu'est-ce qui intéresse le prévisionniste ? Est-ce la précision du débit de pointe prévue, son heure de réalisation, l'avance avec laquelle on sait qu'un seuil de débordement sera atteint ? On peut toujours choisir un critère et une présentation qui mette en valeur la qualité d'un réglage. Il faudrait pouvoir demander aux utilisateurs s'ils sont satisfaits ou non du modèle qui leur a été proposé. 